\title{
BMJ Open Association of physical activity with cognition, metacognition and academic performance in children and adolescents: a protocol for systematic review and meta-analysis
}

\author{
Celia Álvarez-Bueno, ${ }^{1}$ Caterina Pesce, ${ }^{2}$ Iván Cavero-Redondo, ${ }^{1}$ \\ Mairena Sánchez-López, ${ }^{1,3}$ María Jesús Pardo-Guijarro, ${ }^{1,4}$ \\ Vicente Martínez-Vizcaíno ${ }^{1,5}$
}

To cite: Álvarez-Bueno C, Pesce C, Cavero-Redondo I, et al. Association of physical activity with cognition, metacognition and academic performance in children and adolescents: a protocol for systematic review and metaanalysis. BMJ Open 2016;6: e011065. doi:10.1136/ bmjopen-2016-011065

- Prepublication history and additional material is available. To view please visit the journal (http://dx.doi.org/ 10.1136/bmjopen-2016011065).

Received 6 January 2016 Revised 9 May 2016 Accepted 7 June 2016

CrossMark

For numbered affiliations see end of article.

Correspondence to Professor Vicente Martínez Vizcaíno; Vicente.Martinez@uclm.es

\section{ABSTRACT}

Introduction: Schools provide a relevant context for improving children's and adolescents' physical and mental health by increasing physical activity during school hours and/or beyond. The interest in the relationship between physical activity programmes and cognition during development has recently increased, with evidence suggesting a positive association. We present a protocol of systematic reviews and metaanalysis of intervention studies that, by determining the effects of chronic physical exercise on children's and adolescents' cognitive and metacognitive functions, cognitive life skills, academic behaviours and achievement, aims to ensure procedural objectivity and transparency, and maximise the extraction of relevant information to inform policy development.

Methods: This protocol is guided by Preferred Reporting Items for Systematic Review and MetaAnalysis Protocols (PRISMA-P) and by the Cochrane Collaboration Handbook. Databases to be utilised for a thorough selection of the pertinent literature are MEDLINE, EMBASE, Cochrane Central Register of Controlled Trials, Cochrane Database of Systematic Reviews, Web of Science, PsycINFO and ERIC. Selection is proposed to encompass an international and a national publication level, with inclusion of experimental studies written in English or in Spanish, respectively. Also, relevant references included in the selected studies will be considered suitable for review as supplemental sources.

We present an integrated approach to the methodological quality assessment of the selected studies, including the Jadad Scale for the assessment of the quality of randomised controlled trials and the Quality Assessment Tool for Quantitative Studies for pre-post studies and non-randomised controlled trials. The pre-post interventions mean differences will be the primary indicator of the intervention outcome.

Statistical analysis: A subgroup analysis is proposed based on cognitive functions and their neural correlates, metacognitive functions and cognitive life

\section{Strengths and limitations of this study}

- This study presents a comprehensive methodology for analysing the effect of physical activity programmes on main components of children's brain health, cognitive functioning and academic performance that are relevant for policy development.

- Featured in the study is assessment of risk of bias of included studies and heterogeneity among studies with particular reference to individual, task and contextual factors.

- Included in analysis are those factors identified as relevant potential moderators of the relation of physical activity with cognition or academic performance in children and adolescents.

- There is heterogeneity of the assessed outcomes or tests used for assessing the same outcome.

- Generalisation of results constrained by the exclusion of children and adolescents with atypical development is present.

skills, academic achievement areas and academic behaviours.

Trial registration number: PROSPERO CRD42015029913

\section{INTRODUCTION}

In the last decades, scientific evidence on the relationship between chronic physical activity and cognitive/academic performance in childhood and adolescence has attracted increasing attention. ${ }^{1}$ Chronic physical activity interventions have been defined as long lasting repeated bouts of exercise aimed to improve physical fitness. ${ }^{2}$ Chronic physical activity participation has been associated with several mental health benefits in school children, such as improved self-perceptions 
(eg, self-esteem, self-efficacy), emotional regulation (eg, anxiety, depression) and cognitive functioning (eg, information processing, memory, attention). ${ }^{3}$

Owing to the relevant implications for educational policies, many researchers have investigated the effects of chronic physical activity participation on students' success in academic performance at school. ${ }^{4-5}$ The latter is pooled in classroom behaviour (eg, on-task behaviours during learning activities) and academic achievement (eg, school notes and performance in test on school subjects). Attention has also been focused on cognitive executive functions, since their development early in life has been proven predictive of school and lifelong achievement, health and quality of life. ${ }^{6}$ Diamond $^{6}$ distinguishes between core executive functions-inhibitory control, working memory and cognitive flexibility-and higher-level executive functions such as reasoning, planning and problem solving. This higher-level cognition largely overlaps with what is termed metacognition, that is, the ability to supervise and manage cognitive process and to use knowledge to regulate behaviours. ${ }^{2}$

Chronic exercise-cognition research has experienced a progressive shift towards a biochemical and neuroscientific perspective from both, exercise and cognition researchers, ${ }^{7-8}$ as well as developmental neuroscientists. ${ }^{9}$ The positive influence of chronic physical activity has been related to angiogenesis, increasing oxygen saturation and glucose delivery, improving cerebral blood flow and increasing neurotransmitters levels ${ }^{10}$ differences in structural brain volumes, as measured by MRI, ${ }^{11}$ and brain function, as measured by electrical activity recordings. ${ }^{12}$

Previous systematic reviews and meta-analysis have synthetised evidence of the influence of exercise interventions on children's and, less frequently, adolescents' cognition and success in school, focused on quantitative exercise characteristics (intensity, frequency and session duration)..$^{5}{ }^{13-16}$ Of these, one regarded experimental studies only and considered cognitive and psychosocial outcomes jointly. ${ }^{15}$ No study presented results distinguishing between cognitive and metacognitive functions, academic behaviour and achievement. Indeed, this distinction has recently been deemed relevant to understand the potential mediational paths that underlie the relationship between physical activity and academic achievement. $^{2}$

In the search for further mechanisms beyond the neurobiological that may explain the link between chronic physical activity and children's cognition, recent narrative or meta-analytic reviews have focused on the qualitative characteristics of the physical activity interventions. ${ }^{8} 1718$ The difficulty in operationalising the breadth of the exercise quality construct in exercise and cognition research, beyond the mere metabolic and neuromuscular demands of physical exercise tasks, ${ }^{19}$ is a main cause for the underinvestigation of the role played by qualitative exercise characteristics such as coordinative and cognitive task complexity, novelty and diversification, for cognitive development promotion. ${ }^{20}$ Also, developmental neuroscientists interested in interventions aiding children's cognition are increasingly shifting attention towards qualitative forms of physical activity that are not only physically effortful, but also emotionally and socially engaging. ${ }^{9}$ These kinds of interventions often involve physical activities that impinge on core cognitive functions, as well as on a broader range of cognitive skills, such as goal setting, problem solving and self-regulation. ${ }^{1}$ These are cognitive in nature and therefore fall into the field of cognitive sciences, but they are also investigated in psychosocial and socialcognitive research as essential life skills to self-regulate behaviour and successfully adapt it to everyday requirements. ${ }^{21}$ Since they are proven sensitive to designed physical activity interventions, ${ }^{22}$ it has been recently proposed that cognitive life skills may represent a further element to be considered in the relationship between physical activity, cognition and academic behaviour and achievement. ${ }^{8}$

In sum, scientific evidence on the relationship between physical activity and cognitive/academic performance, particularly in regard to its possible moderators and mediators, is still currently insufficient to obtain a comprehensive view that may be useful to inform policies and decision-making. Discrepancies persist regarding the effects of chronic exercise interventions on children's cognition and success in school and life, and reviews still lack consideration of evidence at relevant intersection points between different research areas. Moreover, whereas several narrative and meta-analytic reviews have provided evidence syntheses that are mainly useful for setting future research priorities, they still leave open questions concerning how this evidence can be translated into good practices in ecological settings such as the educational. ${ }^{23}$

Thus, the general aim of the present methodological article is to provide a novel protocol designed to review interventional studies addressing the chronic exercisecognition interaction in children and adolescents for obtaining relevant information for policymakers and decision makers particularly in, but not limited to, the education sector. To this aim, the proposed protocol encompasses different facets of cognitive function and academic performance that, to the best of our knowledge, should be jointly considered to facilitate transitioning evidence of the cognitive benefits of physical activity for children and adolescents into good practices.

\section{OBJECTIVES}

This systematic review and meta-analysis has three objectives: (1) to estimate the effects of chronic physical exercise interventions on different facets of cognitive function and academic performance of children and adolescents; (2) to determine which of those variables benefit most from physical activity; (3) to identify the 
individual, task-related and contextual moderators that may amplify physical activity effects on cognition/academic performance, with particular focus on the qualitative and quantitative characteristics of the physical activity interventions.

Specifically, this systematic review and meta-analysis protocol presents an objective and clear procedure to maximise the extraction of information from experimental studies (randomised controlled trials-RCTs, non-RCTs and controlled pre-post studies), in which data for cognition, metacognition, cognitive life skills, academic behaviour and achievement have been separately or jointly reported as outcomes.

\section{METHODS AND ANALYSIS}

This systematic review and meta-analysis protocol is based on the Preferred Reporting Items for Systematic Review and Meta-Analysis Protocols (PRISMA-P) ${ }^{24}$ and the Cochrane Collaboration Handbook. ${ }^{25}$ This trial has been registered in PROSPERO (registration number: CRD42015029913).

\section{Inclusion/exclusion criteria for study selection \\ Type of studies}

Randomised control trials (RCTs), non-RCTs and controlled pre-post studies written in international language (English) or in the national language (Spanish) of interest.

\section{Type of participants}

Studies assessing the relationship, at developmental age, between chronic physical exercise interventions and cognition, metacognition, cognitive life skills and academic performance variables will be included regardless of sex, weight, ethnicity and socioeconomic status. Studies including participants aged from 4 to 18 years will be considered for inclusion. Among exclusion criteria, the one regarding participants will be the presence of children with any physical condition or any diagnosed disorder of cognition that would impede or limit their ability to participate in school physical activity programmes. If participants are assessed more than once in the same study, data will be extracted and analysed from the different measurements as independent samples.

\section{Type of interventions}

Studies reporting any type of chronic physical exercise intervention, defined as repeated bouts of exercise over time aimed to improve physical fitness and involving multiple sessions over a number of training weeks, months or years, will be eligible for inclusion. Studies reporting the transient effects of single bouts of acute physical exercise will be excluded. Studies comparing different types of chronic physical exercise interventions or examining a chronic physical exercise intervention with or without a control group are considered eligible for inclusion.
Among chronic physical exercise interventions, we will include those defined as: school-based physical exercise interventions, recess time interventions, classroom-based physical activity interventions and extracurricular physical activity interventions. Studies combining physical exercise with other health interventions, such as nutritional interventions, will be excluded when data concerning the effectiveness of physical activity programmes on cognitive or academic performance variables could not be extracted separately.

\section{Type of outcome assessments}

In the attempt to provide a comprehensive view of physical activity effects on the different facets of children's and adolescents' cognition, a broad array of cognitive outcome assessments is warranted, ranging from neural correlates of cognitive functioning to performance measures and observational or self-reported evaluations of cognition, metacognition, cognitive life skills and academic performance.

Indicatively, but not exhaustively, common performance measures for cognitive function assessment are provided by tests such as the Eriksen flanker task, Stroop Colour-Word task, Cognitive Assessment System (CAS), or the Stenberg task. Examples of performance measures for metacognitive function assessment are the Tower of London test and creativity assessment tools such as the Alternate Uses Test. Academic performance assessments regard: (1) academic achievement by curriculum-based marks or specific scales such as the Canadian Achievement Test, Terra Nova test, or Metropolitan achievement test; and (2) academic behaviours by measures such as on-task behaviours, organisation, or attendance. The assessment of cognitive life skills outcomes (goal setting, problem solving, selfregulation) can include self-report measures such as the Life Skills Self Beliefs test, or multisource assessment scales that triangulate self-reports with ratings by significant others (peers, teachers). When cognitive, metacognitive or academic performance outcomes are paralleled by biochemical, brain functional and structural correlates, such measures will also be considered, as they may inform about the biochemical and neural mechanisms underlying physical activity effects on cognition.

\section{Search methods for the identification of studies Electronic search}

The search will be conducted in the MEDLINE, EMBASE, Cochrane Central Register of Controlled Trials, Cochrane Database of Systematic Reviews, Web of Science, PsycINFO and ERIC databases from their inception. Study records will be managed by means of the Mendeley reference manager.

The following search terms (and related truncations, eg, 'cognit' to tap cognition and cognitive) will be used: (1) physical activity, physical education, exercise, fitness, sport; (2) cognition, executive, executive function, $\operatorname{cog}$ nitive control, intelligence, memory, attention, 
metacognition; (3) life skills, goal setting, problem solving, self-regulation; (4) academic, academic achievement, academic grades, academic behaviour, academic performance, classroom behaviour; (5) brain development, brain health, neural, neuroelectric, neurotrophic, neurotrophin, hormone; (6) children, childhood, preschooler, schooler, preadolescent, adolescent, adolescence and (7) trial (see online supplementary appendix I for MEDLINE database search strategy).

Previous reviews and meta-analyses will be checked for additional references and relevant references cited in the selected studies will be screened as supplemental sources.

\section{Data collection and analysis}

Selection of studies

After excluding duplicated records, two reviewers will independently screen titles and abstracts to identify eligible studies according to the inclusion and exclusion criteria. Then, the potential eligible studies will be comprehensively reviewed and their reference list checked for additional relevant studies. Any discrepancies will be resolved by discussion with a third reviewer.

Two authors will independently extract data on publication year, number and age of participants (control and intervention groups), physical exercise intervention characteristics and (meta)cognition/life skills/academic performance variables.

Any disagreement will be resolved by discussion and consensus. If necessary, the authors of the included studies will be contacted to obtain additional relevant information.

\section{Assessment of risk of bias in included studies}

Two researchers will conduct a quality assessment according to the Cochrane Collaboration Handbook. ${ }^{25}$ Any disagreement will be resolved by discussion. A third reviewer will resolve the disagreement if consensus is not reached.

Methodological quality of the RCT will be assessed with the Jadad Scale. ${ }^{26}$ The risk of bias will be evaluated according to three domains: randomisation, double blinding and description of withdrawals and dropouts. Each domain will receive a score of one when the studies satisfy its description. Randomisation will score one extra point if the method to generate the sequence is appropriate. A double blind study will score one extra point if the double blind method is appropriately described. Based on these domains, scores can range from 0 to 5 .

The Quality Assessment Tool for Quantitative Studies $^{27}$ is proposed to assess the quality of pre-post studies and non-RCTs. This tool evaluates seven domains: selection bias, study design, confounders, blinding, data collection method, withdrawals and dropouts. Each domain could be considered strong, moderate or weak, and studies could be classified as strong (with no weak ratings), moderate (with one weak rating) and weak (with two or more weak ratings). If there are insufficient or unclear data describing the required domains, the study authors have to be contacted for more details.

\section{Data synthesis}

Key characteristics and important questions, such as sample size, age of participants, quantitative and qualitative intervention characteristics, and cognitive outcome relevant to the aim of the review, will be summarised in tables (see online supplementary appendix II). Reviewers will determine whether a meta-analysis is possible when data have been extracted. At least five observations addressing the same specific outcome will be required to conduct a meta-analysis.

If it is possible to carry out a meta-analysis, STATA V.13 software will be used to combine the pooled mean differences with $95 \%$ CIs. A fixed-effect model will be used if there is no evidence of heterogeneity; otherwise, a random-effects model will be used. The study heterogeneity will be assessed with an $\mathrm{I}^{2}$ statistic. Usually, $\mathrm{I}^{2}$ values of $<25 \%, 25-50 \%$ and $>50 \%$ represent small, medium and large amounts of heterogeneity, respectively. ${ }^{28}$ Studies with insufficient data to perform the analyses will be omitted from the data synthesis. If there is substantial heterogeneity among the studies and a meta-analysis is not possible, a descriptive analysis will be conducted.

The measure of mean pre-post intervention difference will be the primary indicator of the intervention outcome. Mean differences (standard error (SE)) and standardised mean differences (standard deviation (SD) ) will be calculated for each specific skill or area included in the tests. For example, when the SE is provided, the SD will be calculated according to the following formula: $\mathrm{SD}=\mathrm{SE} \times \sqrt{\mathrm{N}}$. The pooled effect size on the physical intervention and control groups will be compared using the mean differences and SE weight for the number of participants. Last, publication bias will be assessed by means of the method proposed by Egger, as well as visually on a funnel plot. ${ }^{29}$

\section{Subgroup analyses}

Subgroup analyses will be performed based on the main factors that may cause heterogeneity, grouped as individual, task and contextual constraints. Main individual factors that could act as moderators of the exercise-cognition relation are age, weight status and skill level.

Main types of task-related factors are qualitative and quantitative intervention characteristics, the type of cognition assessed and the stability of the intervention outcomes over time. Well-established quantitative parameters of the interventions are intensity, frequency and overall session duration. ${ }^{30}$ The qualitative characteristics of chronic exercise interventions to aid children's and adolescents' cognition have been tentatively classified in different ways in recent reviews. One classification primarily links the physical activity type to its 
specific context of practice: physical education at school, active commuting in the urban route environment and individual versus team sport participation, indoors or outdoors. ${ }^{31}$ Another classification attempts to distinguish studies primarily focused on the metabolic demands of physical activity from those focused on or with deliberate manipulation of the coordinative and cognitive demands: aerobic training, skill-based training, cognitively demanding/enriched physical activity, traditional physical education or combinations of them. ${ }^{18}$

The broad array of facets of cognitive functioning that may be differentially influenced by chronic exercise interventions include: (1) non-executive functions, such as non-verbal ability, spatial ability; (2) core executive functions, which are inhibition, working memory, cognitive flexibility; (3) metacognitive functions (ie, higherlevel executive functions), such as abstract reasoning, planning, problem solving; (4) cognitive life skills, such as goal setting, self-regulation; (5) academic achievement areas, such as mathematics, language, reading, total scores; (6) academic behaviours, such as on-task behaviours, organisation, attendance. Finally, the time of cognitive assessment after intervention cessation influences the effect size and may inform on the outcome stability.

The main contextual factor that may cause heterogeneity of results is the intervention setting: school, out-of-school or laboratory setting. Also, in school-based studies, whether the physical activity intervention enhanced/enriched physical education, classroom-based activity breaks during curricular time, or active play during recess time, must be taken into account.

\section{Sensitivity analysis}

Sensitivity analyses will be conducted excluding studies from the analysis one by one. It needs to be proved that the findings from the meta-analysis are not dependent on arbitrary or unclear decisions. The main argument for carrying out a sensitivity analysis in the present review protocol is the existence of large differences in (1) study design and (2) type of specific assessment tools used. (1) As regards the study design, as indicated under the subheading 'Type of studies', we propose to include RCTs, non-RCTs and control pre-post studies, which may largely differ in their ability to truly tap intervention outcomes. (2) As concerns the specific assessment tools used, they may differ in the extent to which they specifically tap the cognitive function of interest, or may lead to spurious results. For example, academic grades represent final outcome of achievement behaviours that are affected not only by cognitive, but also by motivational, emotional and social factors of the learning context. Furthermore, there also may be differences due to the sensitivity issue, among more narrowly focused cognitive test outcomes. For example, inhibitory control-one of the most commonly studied cognitive functions in developmental exercise-cognition studiesis multifaceted ${ }^{1}$ and has been therefore studied with different tests that tap inhibition of thoughts and memories (eg, Random Number Generation), inhibition as perceptual interference control challenging attention (eg, Eriksen flanker task, Stroop Colour-Word task, expressive attention scale of the Cognitive Assessment System), or inhibition at the behavioural response level (eg, stop-signal task).

\section{DISCUSSION}

A positive association between physical exercise programmes and academic performance has been reported more or less consistently by recent systematic reviews and meta-analyses ${ }^{413-1631}$ that analysed this relationship. The commonality of the abovementioned systematic reviews and meta-analyses is that they all included academic performance outcomes that represent a key variable for policymakers of the education sector. On the other hand, they largely differed as regards other characteristics that must be considered to reach relevant conclusion. Several differences among studies can render the evidence base more or less useful and the take-home message more or less meaningful and generalisable for policymakers. They regard: study design; acute/chronic exercise research type; intervention type and length of follow-up period; type and specificity of outcome measures; type of individual, task-related, and contextual moderators acting on the relationship between physical activity and cognition/ academic performance.

First of all, some reviews ${ }^{13-14} 31$ included not only interventional studies, but also those with cross-sectional or observational designs; this limits the strength of causal conclusions and of the call for more physical activity in school and in out-of-school settings as a means to aid cognitive development and successful academic achievement. A narrative review $^{17}$ that was exclusively focused on interventional research included both, acute and chronic exercise studies, having each of them a different take-home message for policymakers. The transient cognitive benefits of an acute bout of exercise support the call for more physically active breaks interspersed during the sitting learning time and for more physically active academic lessons (eg, 'moved maths'). On the other hand, the cognitive benefits of a longerlasting chronic exercise programme support the call for legislative changes in favour of enhanced physical education and physical activity promotion in out-of-school settings.

To the best of our knowledge, only one systematic review $^{15}$ included a RCT. It considered a broad range of outcomes of aerobic exercise programmes including cognition, academic behaviour and achievement, as well as psychosocial functioning outcomes. However, this review did not provide data for the impact of aerobic exercise programmes on each academic performance component, nor did it include, among the studies with psychosocial outcomes, those regarding physical activity 
effects on cognitive life skills that are linked to successful academic behaviour and achievement.

The lack of separate subgroup analyses of data according to the different academic performance areas or types of cognitive function assessed is common to most of the existing reviews. This limits the possibility to obtain a differentiated view on what type of physical activity interventions work best to reap specific cognitive/academic benefits. Also, the applied conclusions that can be drawn from many of the existing reviews are limited, as explicitly acknowledged, ${ }^{15}$ by the use of different measurement tools and the paucity and diversity of follow-up periods.

The present protocol is aimed at overcoming these limitations by performing subgroup analyses that take into account these issues in combination. Particularly, we follow the call by Tomporowski et $a l^{2}$ to distinguish between cognitive and metacognitive outcomes of physical activity in order to investigate their role in a hypothesised mediational chain linking chronic physical activity and academic performance. The authors state that cognitive assessments in developmental exercise and cognition research prioritise tools that test the cognitive functions of interest during 'on-line' processing. ${ }^{2}$ To explain how exercise impacts children's metacognitive processes and academic performance that develop along a wider time scale, they recommend expanding the view on exercise-cognition relations to encompass cognitive-social factors that underlie the personal awareness of own skills in achieving short-term and long-term goals. The present protocol provides an attempt in this direction, expanding the usual framework for exercisecognition reviews to encompass cognitive life skills and separately analyse cognitive, metacognitive and academic performance outcomes.

As an outlook for future research, $\mathrm{CDC}^{13}$ encouraged analysing the same variables in any given category, to make a summary statement about the magnitude of the effect of physical exercise on academic performance variables. Singh et $a l^{16}$ recognised the inclusion of different study designs and outcome measures as a limitation of conclusions. Tomporowski et $a l^{2}$ recommended improving the information regarding the qualitative and quantitative characteristics of physical activity programmes that enhance children's neurocognitive performance, and to consider the possible moderators and mediators acting on the relationship between chronic exercise and cognition/academic performance during development. Since it is common that moderator variables are included in interventional studies, it will be possible to apply the subgroup and moderation analyses proposed in the Methods section. However, there is still a paucity of studies addressing mediation, ${ }^{8} 3233$ which remains an issue in need of further research before meta-analytic conclusions can be drawn.

The proposed protocol also presents limitations that derive from the deliberate choice of a given trade-off setpoint between inclusion and exclusion criteria to reach relevant conclusions for policymakers of the education and health sectors. Specifically, the broad and heterogeneous array of relevant outcomes that will be included has costs as well as benefits. It offers the possibility to tap different-from biological to behaviouralaspects related to cognitive functioning and academic performance. Nevertheless, the fact that included studies can broadly differ in assessed outcomes or tests used for assessing the same outcome might lead to underestimation of overall effect size, or to highly variable effect sizes among outcome subsets. On the other hand, heterogeneity of studies will be limited regarding participants' characteristics, since studies involving participants with atypical development will be excluded. Nevertheless, in this way, the generalisability of results will be lowered, being traded for a higher comparability of intervention outcomes.

Given the importance of the entire developmental period-from infancy to late adolescence ${ }^{34}$-for brain development and therefore for academic performance, a more detailed and comprehensive view on the exercisecognition relation during development is needed for education and health professionals to orient policy efforts. This protocol provides a clear and structured procedure for maximising the extraction of relevant information and provides summarised information regarding the impact of long-term physical activity programmes on children's cognition and academic performance.

\section{Ethics and dissemination}

Ethical approval will not be needed to apply this review protocol because data will be extracted from published studies and there will be no concerns about privacy. The results of such reviews can be best disseminated by publication in a peer-reviewed journal that broadly reaches researchers interested in hypotheses testing and policymakers interested in the translatability of scientific evidence into good practices.

Developing programmes and strategies to promote physical activity is justified by the international physical activity guidelines, which recommend that children participate in at least $60 \mathrm{~min}$ of daily physical activity. ${ }^{35}$ Nonetheless, the prevalence of overweight children and the total sedentary time that children daily accumulate have risen substantially in the past three decades in most countries. Therefore, schools and communities are encouraged to implement children's physical activity time. However, it is necessary to further our understanding of how to capitalise on physical activity effects on cognitive function and life skills relevant to successful academic performance. The conclusions of the proposed type of systematic review and meta-analysis may support the decisions of school boards, school administrators and policy developers with scientifically grounded arguments on why to maintain or increase the time devoted to curricular or extracurricular physical activity 
and on what type of activities help reap largest cognitive and academic benefits.

\section{Author affiliations}

${ }^{1}$ Universidad de Castilla-La Mancha, Health and Social Research Center, Cuenca, Spain

${ }^{2}$ Department of Movement, Human and Health Sciences, Italian University Sport and Movement "Foro Italico", Rome, Italy

${ }^{3}$ Universidad de Castilla-La Mancha, School of Education, Ciudad Real, Spain

${ }^{4}$ Universidad de Castilla-La Mancha, School of Education, Cuenca, Spain

${ }^{5}$ Facultad de Ciencias de la Salud, Universidad Autónoma de Chile, Talca,

Chile

Contributors VM-V and CA-B designed the study. VM-V was the principal investigator and guarantor. MS-L, CA-B and VM-V were the main coordinators of the study. MS-L, CA-B, MJP-G and IC-R conducted the study. IC-R, CP and MJP-G provided statistical and epidemiological support. VM-V wrote the article with the support of MS-L, CP and CA-B. All the authors revised and approved the final version of the manuscript.

\section{Competing interests None declared.}

Provenance and peer review Not commissioned; externally peer reviewed.

Open Access This is an Open Access article distributed in accordance with the Creative Commons Attribution Non Commercial (CC BY-NC 4.0) license, which permits others to distribute, remix, adapt, build upon this work noncommercially, and license their derivative works on different terms, provided the original work is properly cited and the use is non-commercial. See: http:// creativecommons.org/licenses/by-nc/4.0/

\section{REFERENCES}

1. Howie EK, Pate RR. Physical activity and academic achievement in children: a historical perspective. J Sport Health Sci 2012;1:160-9.

2. Tomporowski PD, McCullick B, Pendleton DM, et al. Exercise and children's cognition: the role of exercise characteristics and a place for metacognition. J Sport Health Sci 2015;4:47-55.

3. Biddle SJH, Asare M. Physical activity and mental health in children and adolescents: a review of reviews. Br J Sports Med 2011;45:886-95.

4. Rasberry CN, Lee SM, Robin L, et al. The association between school-based physical activity, including physical education, and academic performance: a systematic review of the literature. Prev Med (Baltim) 2011;52:S10-20.

5. Donnelly JE, Lambourne K. Classroom-based physical activity, cognition, and academic achievement. Prev Med 2011;52(Suppl 1): S36-42.

6. Diamond A. Executive functions. Annu Rev Psychol 2013;64:135-68.

7. Khan NA, Hillman $\mathrm{CH}$. The relation of childhood physical activity and aerobic fitness to brain function and cognition: a review. Pediatr Exerc Sci 2014;26:138-46.

8. Pesce C, Ben-Soussan D. 'Cogito ergo sum' or 'ambulo ergo sum'? New perspectives in developmental exercise and cognition research In: McMorris T, ed. Exercise-cognition interaction: neuroscience perspectives. Elsevier, 2016.

9. Diamond A, Ling DS. Conclusions about interventions, programs, and approaches for improving executive functions that appear justified and those that, despite much hype, do not. Dev Cogn Neurosci 2016;18:34-48.

10. Diamond $A B$. The cognitive benefits of exercise in youth. Curr Sports Med Rep 2015;14:320-6.

11. Chaddock-Heyman L, Hillman $\mathrm{CH}$, Cohen $\mathrm{NJ}$, et al. The importance of physical activity and aerobic fitness for cognitive control and memory in children. Monogr Soc Res Child Dev 2014;79:25-50.

12. Hillman $\mathrm{CH}$, Pontifex MB, Castelli DM, et al. Effects of the FITKids randomized controlled trial on executive control and brain function. Pediatrics 2014;134:e1063-71.
13. Centers for Disease Control and Prevention (CDC). The association between school-based physical activity, including physical education, and academic performance. Atlanta, GA: US. Department of Health and Humans Services, 2010.

14. Fedewa AL, Ahn S. The effects of physical activity and physical fitness on children's achievement and cognitive outcomes: a meta-analysis. Res Q Exerc Sport 2011;82:521-35.

15. Lees C, Hopkins J. Effect of aerobic exercise on cognition, academic achievement, and psychosocial function in children: a systematic review of randomized control trials. Prev Chronic Dis 2013;10:E174.

16. Singh A, Uijtdewilligen L, Twisk JWR, et al. Physical activity and performance at school: a systematic review of the literature including a methodological quality assessment. Arch Pediatr Adolesc Med 2012;166:49-55.

17. Tomporowski PD, Lambourne K, Okumura MS. Physical activity interventions and children's mental function: an introduction and overview. Prev Med 2011;52(Suppl 1):S3-9.

18. Vazou S, Pesce $C$, Lakes K, et al. More than one road leads to Rome: a narrative review and meta-analysis of physical activity intervention effects on children's cognition. Int J Sport Exerc Psychol In press.

19. Pesce C. Shifting the focus from quantitative to qualitative exercise characteristics in exercise and cognition research. J Sport Exerc Psych 2012;34:766-86.

20. Pesce C, Croce R, Ben-Soussan TD, et al. Variability of practice as an interface between motor and cognitive development promotion. Int J Sport Exerc Psychol In press.

21. Hodge K, Danish S, Martin J. Developing a conceptual framework for life skills interventions. Couns Psychol 2012;20: $1-28$.

22. Goudas M. Prologue: a review of life skills teaching in sport and physical education. Hellenic J Psychol 2010;7:241-58.

23. Kriemler S, Meyer U, Martin E, et al. Effect of school-based interventions on physical activity in children and adolescents: a review of reviews and systematic update. Br J Sports Med 2011;45:923-30.

24. Moher D, Shamseer L, Clarke M, et al. Preferred reporting items for systematic review and meta-analysis protocols (PRISMA-P) 2015 statement. Syst Rev 2015;4:1.

25. Higgins JP, Green S, eds. Cochrane handbook for systematic reviews of interventions. 5.1.0 [Updated March 2011]. The Cochrane Collaboration, 2011. http:/handbook.cochrane.org.

26. Jadad AR, Moore RA, Carroll D, et al. Assessing the quality of reports of randomized clinical trials: is blinding necessary? Control Clin Trials 1996;17:1-12.

27. National Collaborating Centre for Methods and Tools. Quality assessment tool for quantitative studies. Hamilton, ON: McMaster University, 2008 [Updated 13 April, 2010].

28. Higgins JPT, Thompson SG. Quantifying heterogeneity in a meta-analysis. Stat Med 2002;21:1539-58.

29. Sterne JA, Egger M, Smith GD. Systematic reviews in health care: investigating and dealing with publication and other biases in meta-analysis. BMJ 2001;323:101-5.

30. Tremblay MS, LeBlanc AG, Carson V, et al. Canadian physical activity guidelines for the early years (aged 0-4 years). Appl Physiol Nutr Metab 2012;37:345-56.

31. Esteban-Cornejo I, Tejero-Gonzalez CM, Sallis JF, et al. Physical activity and cognition in adolescents: a systematic review. J Sci Med Sport 2015;18:534-9.

32. Lambourne K, Hansen DM, Szabo AN, et al. Indirect and direct relations between aerobic fitness, physical activity, and academic achievement in elementary school students. Ment Health Phys Act 2013;6:165-71.

33. Pesce C, Masci I, Marchetti R, et al. Deliberate play and preparation jointly benefit motor and cognitive development: mediated and moderated effects. Front Psychol 2016;7:349.

34. Andersen SL. Trajectories of brain development: point of vulnerability or window of opportunity? Neurosci Biobehav Rev 2003;27(1):3-18.

35. Ekelund U, Luan JA, Sherar LB, et al. Moderate to vigorous physical activity and sedentary time and cardiometabolic risk factors in children and adolescents. JAMA 2012;307:704-12. 